\title{
Gender difference of Caller in Successful Dispatcher assisted Cardiopulmonary resuscitation in Out-of-hospital Cardiac arrest.
}

MW Kim, TH Kim, SD Shin, KJ Song, YJ Kim, KJ Hong, WP Hong, YS Ro, Joo Jung, JH Park, HH Ryu, HW Ryu Emergency department, Seoul National University Hospital Emergency department, Kyungpook National University Hospital Emergency department, Chonnam National University Hospital

\section{Objectives}

The effects of bystander characteristic on successful bystander CPR is not known. The aim of this study is to evaluate the bystander characteristics and the effect of gender of bystander on successful bystander CPR.

\section{Methods}

Subject

Nationwide OHCA registry in Korea(2014 2017)

Adult OHCA patients with presumed cardiac etiology

Cases that the callers got CPR instruction via telephone by dispatchers

\section{Exclusion criteria}

Less than 18 years old

OHCA detected by EMS personnel

Without information of the caller

\section{Exposure}

The Gender of the caller(Male/Female)

\section{Main outcomes}

Successful bystander CPR assessed by the dispatcher Hospital mortality and Neurological recovery

\section{Statistical analysis}

- Descriptive statistic for patients and caller characteristics - Multivariable logistic regression analysis : Calculate adjusted odds ratios (AORs) with 95\% confidence intervals (95\% CIs) for male caller compared to female caller / Adjusting for patients' age, gender, past medical history, Location of arrest, witnessed arrest, ECG, Case year, metropolitan city, CPR education status - Interaction model (Patient gender*aller gender) added to compare the effect size of the hypoxia across shock

- Sensitivity analysis (Location of arrest)

Table 1. Demographic characteristics of study population by gender of caller

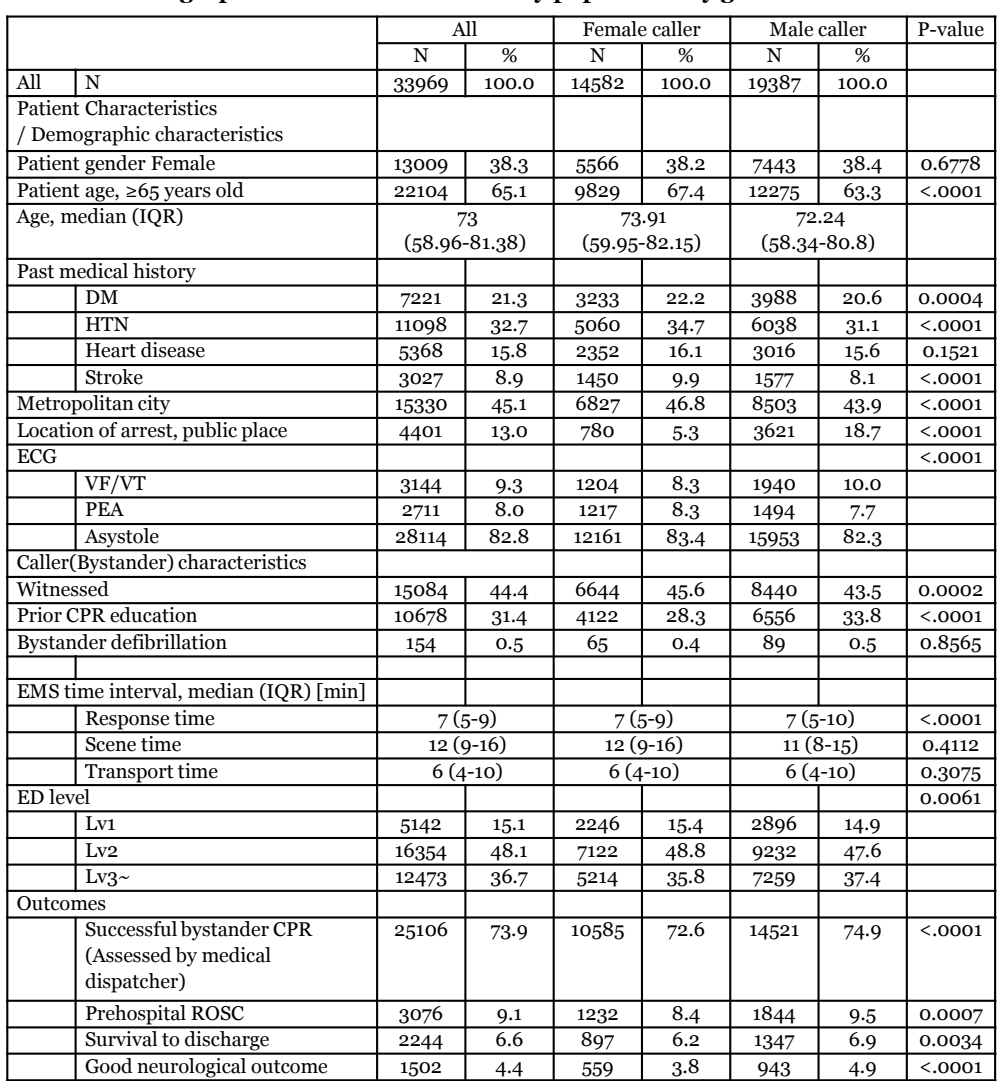

Figure 1. Patient flow

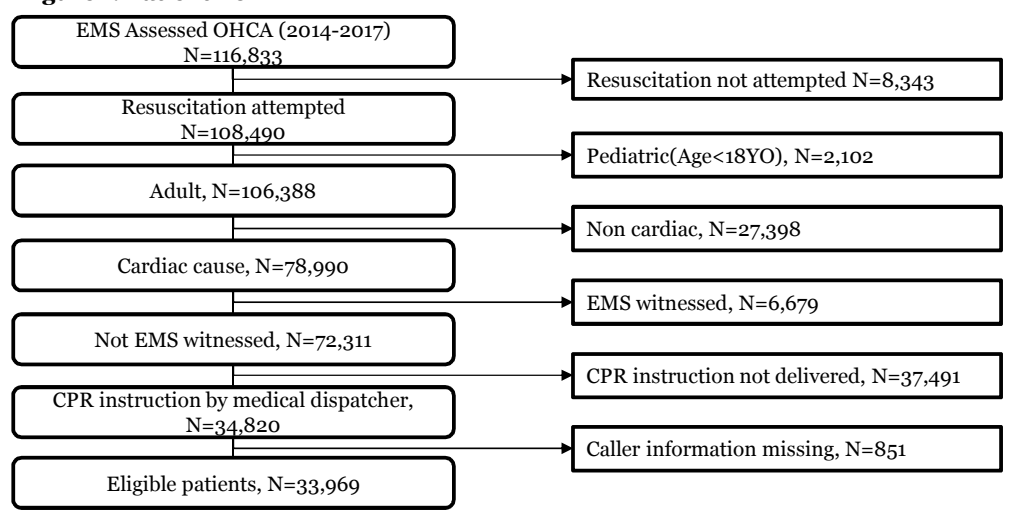

Table 2. Time to CPR by gender of caller

\begin{tabular}{|c|c|c|c|c|c|c|c|}
\hline & All & & Female & & Male ce & & P-value \\
\hline & $\mathrm{N}$ & $\%$ & $\mathrm{~N}$ & & & $\%$ & \\
\hline $\begin{array}{l}\text { Call to medical dispatcher time, } \\
\text { median (IQR) [sec] ( } \mathrm{N}=33969)\end{array}$ & 50( & -73) & & & & -73) & \\
\hline $\begin{array}{l}\text { Call to CPR time, } \\
\text { median (IQR) [sec] (N=30178) }\end{array}$ & $171(1$ & -234) & 176( & 239） & 168( & -231) & \\
\hline $\begin{array}{l}\text { Medical Dispatcher to CPR time, } \\
\text { median (IQR) [sec] }(\mathrm{N}=32580)\end{array}$ & $111(7$ & $-164)$ & 115 & 169) & 109 & $-160)$ & \\
\hline Call to CPR time $<180 \mathrm{~s}$ & 16239 & 53.8 & 6714 & 51.8 & 9525 & $55 \cdot 3$ & $<.0001$ \\
\hline $\begin{array}{l}\text { Medical dispatcher Time } \\
\text { to bystander } \mathrm{CPR}<6 \mathrm{OS}\end{array}$ & 5388 & 16.5 & 2231 & 16.0 & 3157 & 16.9 & 0.0247 \\
\hline
\end{tabular}

Table 3. Multivariable logistic regression for male caller compared to female caller

\begin{tabular}{|c|c|c|c|c|c|c|c|c|c|c|}
\hline \multirow{3}{*}{\multicolumn{2}{|c|}{\begin{tabular}{c|c} 
& Outcomes \\
\cline { 2 - 2 } & $\mathrm{n} / \mathrm{N}(\%)$ \\
Successful bystander CPR & \\
\end{tabular}}} & \multicolumn{3}{|c|}{ Model 1} & \multicolumn{3}{|c|}{ Model2* } & \multicolumn{3}{|c|}{ Model $3^{* *}$} \\
\hline & & \multirow[t]{2}{*}{$\overline{\mathrm{AOR}}$} & \multicolumn{2}{|c|}{$95 \% \mathrm{CIs}$} & \multirow[t]{2}{*}{$\mathrm{AOR}$} & \multicolumn{2}{|c|}{$95 \% \mathrm{CIs}$} & \multirow{2}{*}{ AOR } & \multicolumn{2}{|c|}{$95 \% \mathrm{CIs}$} \\
\hline & & & & & & & & & & \\
\hline \begin{tabular}{l|l} 
& Female callder \\
\end{tabular} & $10585 / 14582(72.6)$ & 1.00 & & & 1.00 & & & 1.00 & & \\
\hline \begin{tabular}{l|l} 
Male caller \\
\end{tabular} & $14521 / 19387(74.9)$ & 1.12 & 1.06 & 1.17 & 1.12 & 1.07 & 1.18 & 1.17 & 1.12 & 1.24 \\
\hline \multicolumn{11}{|l|}{ Survival to discharge } \\
\hline \begin{tabular}{l|l} 
Female caller \\
\end{tabular} & $897 / 14582(6.2)$ & 1.00 & & & 1.00 & & & 1.00 & & \\
\hline \begin{tabular}{l|l|} 
Male caller \\
\end{tabular} & $1347 / 19387(6.9)$ & 1.10 & 1.01 & 1.20 & 1.12 & 1.02 & 1.22 & 0.85 & 0.76 & 0.96 \\
\hline \multicolumn{11}{|c|}{ Good neurologic recovery } \\
\hline Female caller & $559 / 14582(3.8)$ & 1.00 & & & 1.00 & & & 1.00 & & \\
\hline Male caller & $943 / 19387(4.9)$ & 1.25 & 1.12 & 1.39 & 1.26 & 1.13 & 1.41 & 0.93 & 0.80 & 1.08 \\
\hline
\end{tabular}

Model 1. adjusted for patient's age and gender

*Model 2 adjusted for patient's age and gender, comorbidities(Hypertension, Heart diseases, Stroke)

${ }^{*}$ Model 3.adjusted for patient's age and gender, comorbidities(Hypertension, Heart diseases, Stroke) + Arrest \& caller characteristic

Table 4. Interaction analysis (Patient gender $x$ caller gender)

\begin{tabular}{|c|c|c|c|}
\hline & $\begin{array}{c}\text { Successful bystander } \\
\text { CPR }\end{array}$ & Survival to discharge & $\begin{array}{l}\text { Good neurological } \\
\text { recovery }\end{array}$ \\
\hline & Adjusted OR $(95 \% \mathrm{CI})$ & Adjusted OR $(95 \% \mathrm{CI})$ & Adjusted OR $(95 \% \mathrm{CI})$ \\
\hline \multicolumn{4}{|l|}{ Female Patient } \\
\hline \begin{tabular}{l|l} 
Female caller \\
\end{tabular} & 1.00 & 1.00 & 1.00 \\
\hline \begin{tabular}{|l|l|} 
& Male caller \\
\end{tabular} & $0.97(0.89-1.05)$ & $0.82(0.66-1.03)$ & $1.05(0.76-1.45)$ \\
\hline \multicolumn{4}{|l|}{ Male Patient } \\
\hline \begin{tabular}{l|l} 
Female caller \\
\end{tabular} & 1.00 & 1.00 & 1.00 \\
\hline Male caller & $1.33(1.25-1.42)$ & $0.87(0.75-0.99)$ & $0.90(0.76-1.06)$ \\
\hline
\end{tabular}

Table 5. Sensitivity analysis : Location of arrest

\begin{tabular}{|c|c|c|c|c|}
\hline & Outcomes & \multicolumn{3}{|c|}{ Model3 } \\
\hline & $\mathrm{n} / \mathrm{N}(\%)$ & AOR & \multicolumn{2}{|c|}{$95 \% \mathrm{CI}$} \\
\hline \multicolumn{5}{|c|}{ Public } \\
\hline \multicolumn{5}{|c|}{ Successful bystander CPR } \\
\hline \begin{tabular}{l|l|} 
& Female caller \\
\end{tabular} & $552 / 780(70.8)$ & 1.00 & & \\
\hline \begin{tabular}{|l|} 
Male caller \\
\end{tabular} & $2659 / 3621(73.4)$ & 1.14 & 0.95 & 1.36 \\
\hline \multicolumn{5}{|l|}{ Survival to discharge } \\
\hline \begin{tabular}{l|l} 
& Female caller \\
\end{tabular} & $114 / 780(14.6)$ & 1.00 & & \\
\hline \begin{tabular}{|l|l|} 
& Male caller \\
\end{tabular} & $663 / 3621(18.3)$ & 1.12 & 0.83 & 1.50 \\
\hline \multicolumn{5}{|c|}{ Good neurologic recovery } \\
\hline \begin{tabular}{l|l} 
& Female caller \\
\end{tabular} & $86 / 780(11.0)$ & 1.00 & & \\
\hline Male caller & $505 / 3621(13.9)$ & 0.98 & 0.70 & 1.36 \\
\hline \multicolumn{5}{|l|}{ Private } \\
\hline \multicolumn{5}{|c|}{ Successful bystander CPR } \\
\hline \begin{tabular}{l|l} 
& Female caller \\
\end{tabular} & $10033 / 13802(72.7)$ & 1.00 & & \\
\hline \begin{tabular}{l|l} 
& Male caller \\
\end{tabular} & $11862 / 15766(75.2)$ & 1.18 & 1.12 & 1.24 \\
\hline \multicolumn{5}{|l|}{ Survival to discharge } \\
\hline \begin{tabular}{l|l} 
& Female caller \\
\end{tabular} & $783 / 13802(5.7)$ & 1.00 & & \\
\hline \begin{tabular}{l|l} 
Male caller \\
\end{tabular} & $684 / 15766(4 \cdot 3)$ & 0.81 & 0.71 & 0.92 \\
\hline \multicolumn{5}{|c|}{ Good neurologic recovery } \\
\hline \begin{tabular}{l|l} 
Female caller \\
\end{tabular} & $473 / 13802(3.4)$ & 1.00 & & \\
\hline Male caller & $438 / 15766(2.8)$ & 0.92 & 0.78 & 1.10 \\
\hline
\end{tabular}

\section{Conclusion}

Male bystanders showed a higher success rate on bystander CPR than female bystander but showed no higher survival or neurologic recovery. Different education and instruction protocols by dispatcher according to the bystander gender would be needed to improve bystander CPR rate. 